\title{
TOTAL POSITIVITY OF FUNDAMENTAL SOLUTIONS OF PARABOLIC EQUATIONS
}

\author{
SAMUEL KARLIN AND JAMES MCGREGOR ${ }^{1}$
}

Introduction. A real-valued function $p(x, y)$ defined for $-\infty \leqq s_{1}<x$, $y<s_{2} \leqq+\infty$ is called totally positive if all of the "minors"

$$
p\left(\begin{array}{l}
x_{1}, x_{2}, \cdots, x_{n} \\
y_{1}, y_{2}, \cdots, y_{n}
\end{array}\right)=\operatorname{det} p\left(x_{i}, y_{j}\right)
$$

where $s_{1}<x_{1}<x_{2}<\cdots<x_{n}<s_{2}, s_{1}<y_{1}<y_{2}<\cdots<y_{n}<s_{2}$ and $n=1,2, \cdots$ are non-negative. The kernel is called strictly totally positive if all such minors are strictly greater than zero.

We are particularly interested in the kernel

$$
p(t ; x, y)=(4 \pi t)^{-1 / 2} \exp \left\{-(x-y)^{2} / 4 t\right\},
$$

$s_{1}=-\infty, s_{2}=+\infty$, and generalizations of it. For any fixed $t>0$ this kernel is strictly totally positive, as is well known [4]. Now (1) is the fundamental solution of the heat equation on the real line, and the generalization we are aiming for is to replace (1) by the fundamental solution of a more general heat equation

$$
\frac{\partial p}{\partial t}=\frac{1}{\rho(x)} \frac{\partial}{\partial x}\left(\kappa(x) \frac{\partial p}{\partial x}\right)-q(x) p,
$$

where $\rho, \kappa$ and $q$ are positive functions, $-\infty \leqq s_{1}<x<s_{2} \leqq+\infty$, and an appropriate boundary condition at each end of the interval is specified. Any such fundamental solution of (2) may be interpreted as the transition probability density function of a diffusion process, and should therefore be totally positive according to a probabilistic theorem of the authors [1]. We give below a nonprobabilistic and quite general proof of this fact. A number of important cases of (2) have already been analyzed [2] by the authors using a variety of special devices.

1. Hypotheses. Rather than treat (2) we will consider the more general differential equations introduced by Feller, see McKean [3] and the bibliography given there. Let $S=\left(s_{1}, s_{2}\right)$ be an open, not necessarily finite interval on the real line with $s_{1}<0<s_{2}$. Let $m(\cdot)$ and $k(\cdot)$ be non-negative Borel measures on $S$, finite on compact sets, with $m$ strictly positive on open subsets of $S$. Let $D$ be the collection

Received by the editors October 26, 1960.

1 This work was supported by the National Science Foundation. 
of all real valued continuous functions $f$ on $S$ such that the one sided derivative

$$
f^{+}(x)=\lim _{h \rightarrow 0+} \frac{f(x+h)-f(x)}{h}
$$

exists for each $x$ in $S$ and

$$
A f=\frac{f^{+}(d x)-f(x) k(d x)}{m(d x)}
$$

is continuous on $S$. The meaning of $\left(^{*}\right)$ is that for $s_{1}<a<b<s_{2}$

$$
f^{+}(b)-f^{+}(a)=\int_{(a, b]} A f d m+\int_{(a, b]} f d k .
$$

The mapping $f \rightarrow A f$ determines a linear transformation of $D$ into continuous functions on $S$. Our general heat equation is

$$
\frac{\partial p}{\partial t}=A p
$$

By an appropriate change of scale, (2) may be viewed as a special case of (3). As a matter of fact (3) is slightly more general than the equation treated by McKean [3]. However, we are going to use the results of McKean, which are known to be valid for (3), as presumably will be shown in the forthcoming book by Ito and McKean.

We impose a pair of classical unmixed boundary conditions such as

$$
\begin{aligned}
& p_{1} u\left(s_{1}\right)-\left(1-p_{1}\right) u^{+}\left(s_{1}\right)=0 \\
& p_{2} u\left(s_{2}\right)+\left(1-p_{2}\right) u^{-}\left(s_{2}\right)=0
\end{aligned}
$$

with $0 \leqq p_{1}, p_{2} \leqq 1$. The precise nature of the allowable boundary conditions at $s_{i}$ depends on the nature of the boundary, and for a discussion of this we refer to McKean [3, p. 522].

2. The resolvent. We now quote a number of results from McKean's paper. The differential equation

$$
A u=\lambda u
$$

has for every $\lambda>0$ a pair of solutions $u_{1}(x, \lambda), u_{2}(x, \lambda)$ defined for $x \in S$ such that $u_{1}$ is positive, strictly increasing, and satisfies the boundary condition at $x=s_{1}$, while $u_{2}$ is positive, strictly decreasing, and satisfies the boundary condition at $x=s_{2}$. Moreover the Wronskian $u_{1}^{+} u_{2}-u_{1} u_{2}^{+}$is a positive constant which can be taken to be one. It is shown by McKean that the resolvent kernel 


$$
G(x, y ; \lambda)= \begin{cases}u_{1}(x, \lambda) u_{2}(y, \lambda), & x \leqq y, \\ u_{2}(x, \lambda) u_{1}(y, \lambda), & x \geqq y,\end{cases}
$$

can be iterated and satisfies $(\lambda>0)$

$$
\frac{\partial^{n}}{\partial \lambda^{n}} G(x, y ; \lambda)=(-1)^{n} n ! G_{n+1}(x, y ; \lambda)
$$

where $G_{1}(x, y ; \lambda)=G(x, y ; \lambda)$ and

$$
G_{n+1}(x, y ; \lambda)=\int_{S} G_{n}(x, z ; \lambda) G(z, y ; \lambda) m(d z) .
$$

Now it is well known that a kernel of the form (4), with $\lambda$ fixed and in which $u_{1}, u_{2}$ are positive and the ratio $u_{1} / u_{2}$ is strictly increasing, is a totally positive kernel. The arguments for this in the case of a finite matrix of similar structure are due to Gantmacher and Krein [6]. The typical minor of the second iterate of $G$ is expressed as

$$
\begin{aligned}
& G_{2}\left(\begin{array}{l}
x_{1}, \cdots, x_{n} \\
y_{1}, \cdots, y_{n}
\end{array} ;\right) \\
& \quad=\int_{\xi_{1}<\cdots<\xi_{n}}^{\ldots} \int G\left(\begin{array}{l}
x_{1}, \cdots, x_{n} \\
\left.\xi_{1}, \cdots, \lambda\right) G\left(\begin{array}{l}
\xi_{1}, \cdots, \xi_{n} \\
y_{1}, \cdots, y_{n} ; \lambda
\end{array}\right) \\
\cdot m\left(d \xi_{1}\right) \cdots m\left(d \xi_{n}\right)
\end{array}\right.
\end{aligned}
$$

which readily generalizes to higher order iterates. The integrand in (6) is non-negative and this provides the tool for showing that for fixed $\lambda>0$ the iterates of $G$ are totally positive.

3. The fundamental solution. It is shown by McKean that (3) possesses a unique fundamental solution $p(t ; x, y)$ satisfying the given boundary conditions and that

$$
\int_{0}^{\infty} e^{-\lambda t} p(t ; x, y) d t=G(x, y ; \lambda), \quad \lambda>0 .
$$

Moreover $p(t ; x, y)$ is continuous on $(0, \infty) \times S \times S$. Hence by the real inversion formula for the Laplace transform (Widder [5, p. 288]) we have

$$
\begin{aligned}
p(t ; x, y) & =\lim _{k \rightarrow \infty} \frac{(-1)^{k}}{k !}\left(\frac{k}{t}\right)^{k+1}\left[\frac{\partial^{k}}{\partial \lambda^{k}} G(x, y ; \lambda)\right]_{\lambda-k / t} \\
& =\lim _{k \rightarrow \infty}\left(\frac{k}{t}\right)^{k+1} G_{k+1}\left(x, y ; \frac{k}{t}\right) .
\end{aligned}
$$


For $k \geqq 1$ and each fixed $t>0$ we pointed out above that the kernel $q_{k}(x, y)=(k / t)^{k+1} G_{k+1}(x, y ; k / t)$ is totally positive and hence $p(t ; x, y)$, as a pointwise limit of such kernels, is likewise totally positive. This proves the following.

TheOREM. The fundamental solution of (3) subject to a classical boundary condition at each end of the interval $S$ is for every fixed $t>0$ a totally positive kernel.

\section{REFERENCES}

1. S. Karlin and J. McGregor, Coincidence probabilities, Pacific J. Math. 9 (1959), 1141-1164.

2. - Classical diffusion processes and total positivity, J. Math. Anal. Appl. 1 (1960), 163-183.

3. H. P. McKean, Jr., Elementary solutions for certain parabolic partial differential equations, Trans. Amer. Math. Soc. 82 (1956), 519-548.

4. I. J. Schoenberg, On smoothing operations and their generating functions, Bull. Amer. Math. Soc. 59 (1953), 199-230.

5. D. Widder, The Laplace transform, Princeton Univ. Press, Princeton, N. J., 1941.

6. F. Gantmacher and M. Krein, Sur les matrices completement non negatives et oscillatoires, Compositio Math. 4 (1937), 445-476.

STANFORD UNIVERSITY 